\title{
High-Performance Computational Electromagnetic Methods Applied to the Design of Patch Antenna with EBG Structure
}

\author{
R. C. Hadarig, M. E. de Cos, and F. Las-Heras \\ Area de Teoría de la Señal y Comunicaciones, Departamento de Ingeniería Eléctrica, Universidad de Oviedo, Edificio Polivalente, \\ Modulo 8, Campus Universitario de Gijón, 33203 Gijón, Spain \\ Correspondence should be addressed to M. E. de Cos, medecos@tsc.uniovi.es
}

Received 14 June 2011; Revised 19 September 2011; Accepted 20 September 2011

Academic Editor: Shyh-Kang Jeng

Copyright ( 92012 R. C. Hadarig et al. This is an open access article distributed under the Creative Commons Attribution License, which permits unrestricted use, distribution, and reproduction in any medium, provided the original work is properly cited.

In this contribution High-Performance Computing electromagnetic methods are applied to the design of a patch antenna combined with EBG structure in order to obtain bandwidth enhancement. The electrical characteristics of the embedded structure (patch antenna surrounded by EBG unit cells) are evaluated by means of method of moment technique (MoM) whereas for designing the unit cell, the finite element method (FEM) together with the Bloch-Floquet theory is used. The manufactured prototypes are characterized in terms of return loss and radiation pattern in an anechoic chamber.

\section{Introduction}

Microstrip patch antennas, which are used for both defense and commercial applications, are replacing many conventional notprinted antennas. The most important properties of microstrip patch antennas are the lightweight, smallvolume and the mass production at low cost whereas intrinsic disadvantages that limit their applications are low gain, narrow bandwidth, and excitation of surface waves [1]. In order to design antennas with better efficiency and gain and lower backlobe and sidelobe levels, EBG structures can be used [2-7]. In previous works [8-16] several narrow band antennas have been mounted on EBG structures. In this contribution, for obtaining bandwidth enhancement in the $2.48 \mathrm{GHz}$ band, the band-gap of the EBG lattice is designed to be adjacent to the frequency band of the patch antenna, so that when integrating together both structures their resonances couple each other and as a result a wider bandwidth will be generated. The final design is uniplanar and in addition there is no need for via holes.

The aim of this work is challenging because two resonant structures are involved and when integrated together their resonant behavior is mutually influenced. For this reason the precision in the simulation results is a key point to achieve bandwidth enhancement. At this point is where highperformance computational electromagnetics play a funda- mental roll in order to get a proper design in a reasonable time.

\section{Microstrip Antenna}

The antenna design has been carried out using Method of Moments (Momentum) electromagnetic simulator [17] and its geometry (Figure 1(a)) was optimized varying the values in a continuous range in order to obtain the frequency band of interest $(2.48 \mathrm{GHz})$ and the minimum bandwidth requested (the process is an iterative one in which there will be a tradeoff between the variation of antenna's geometry and its effects on the performance). Using parameter sweep with MoM in a 2 core Intel Xeon X5560 48 GB RAM (equivalent to 16 execution threads) server, there can be obtained the solution for 81 different values of the swept parameter in just one minute. This is due to a mesh definition so that 20 cells per wavelength at $3 \mathrm{GHz}$ are taken which leads to 249 rectangular cells and 171 triangular cells with a matrix size of 740 . After applying mesh reduction a matrix size of 610 results. A dielectric substrate, ROGER3010 having $1.27 \mathrm{~mm}$ thickness, $\varepsilon_{r}=10.2$ relative dielectric permittivity, and loss tangent of 0.0023 , has been used. The measured operating bandwidth of the patch antenna is $23 \mathrm{MHz}$ (Figure 4). The difference in bandwidth between the simulated $(20 \mathrm{MHz})$ and measured $(23 \mathrm{MHz})$ results could be 


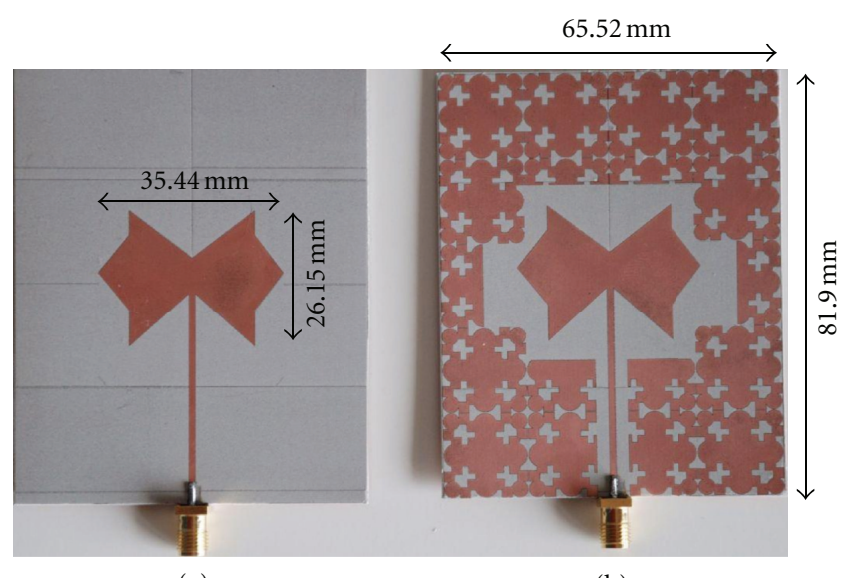

(a)

(b)

Figure 1: Manufactured prototypes: (a) patch antenna (b) patch antenna-EBG.

due to the fact that the commercial MoM software considers infinite extension for the dielectric substrate, or even more likely due to manufacturing tolerances.

\section{EBG Characterization}

The unit cell of an EBG lattice consists of metal pads and sometimes narrow lines that implement a distributed LC circuit having a resonant frequency [18]. In [19] the same unit cell but in different frequency band is characterized from the point of view of an AMC, computing the phase of the reflection coefficient on its surface, from an uniform incident plane wave. To search for the frequency band in which the periodic structure shows the AMC behavior, the finite element method (FEM) together with the Bloch-Floquet theory is used. A single cell of the lattice (with periodic boundary conditions (PBCs) on its four sides) is simulated to model an infinite structure. The unit cell dimensions are $W \times W=16.38 \times 16.38 \mathrm{~mm}^{2}$. The simulation is carried out using a server with 2 processors Intel Xeon E5620 and 64 GB RAM in a configuration equivalent to 2 execution threats and 32 GB RAM. The air-box size is $\lambda / 2$ at the lower frequency considered in the simulation (which is $1 \mathrm{GHz}$ ). The solution frequency is $3 \mathrm{GHz}$ and a frequency sweep is carried out from $1 \mathrm{GHz}$ to $3 \mathrm{GHz}$ with a $0.01 \mathrm{GHz}$ frequency step. The mesh is established to take at least 10 tetrahedra per wavelength at the solution frequency $(3 \mathrm{GHz})$. Mixed-order basis functions and $30 \%$ lambda refinement are used. Maximum Delta S is fixed to 0.02 for the S-parameter calculations. All this leads to a mesh with 3642 tetrahedra (2889 for the air-box and 753 for the substrate), a matrix size of 16101, and a computational time of 8 minutes and 44 seconds for the aforementioned frequency sweep.

However, for the intended application, the structure should show EBG behavior. Even though the finite element method (FEM) uses specific boundary conditions such as simulating just half the volume under study (using Perfect Magnetic Conductor (PMC) boundary condition in one of the volume walls, the one that would cut the volume in two identical parts, the size of the electromagnetic problem

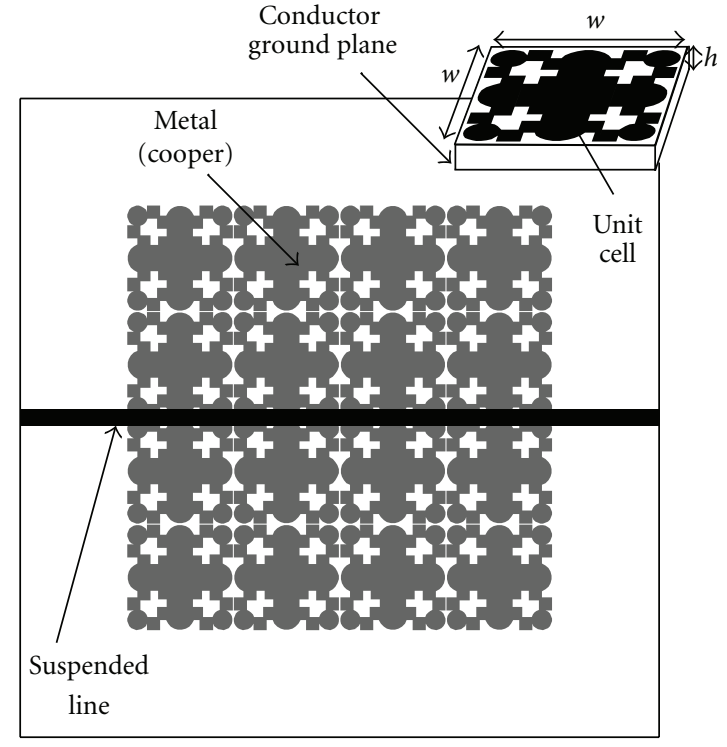

FIGURE 2: Schematic of suspended line above EBG surface (top view).

that needs to be simulated is reduced), for the current scenario the method of moments (MoM) could yield a faster computational time (using MoM only the conductive structure is meshed whereas using FEM, the whole volume around the structure including the air box is meshed). The periodic structure can be characterized as EBG using the suspended strip method $[20,21]$ and so the transmission coefficient $\left(S_{21}\right)$ of a suspended strip line over the periodic structure is simulated (Figure 2). The structure will block the transmission of power along the strip line for frequencies within the band-gap region and a noticeable reduction of $S_{21}$ can be observed at a certain frequency band. To accomplish this simulation the mesh has been defined so that 20 cells per wavelength at $3 \mathrm{GHz}$ are taken leading to 2085 rectangular cells and 5008 triangular cells with a matrix size of 9706 . After applying mesh reduction results a matrix size of 2352 . The simulation time for 25 frequency steps is 50 minutes in a 2core Intel Xeon X5560 48 GB RAM (equivalent to 16 threads execution) server.

The band-gap of the EBG structure $(45 \mathrm{MHz})$ is adjacent to the bandwidth of the patch antenna (see Figure 3 ); thus when combining the two structures together, bandwidth enhancement is obtained without disturbing other characteristics of the patch antenna, such as the radiation pattern.

\section{Patch Antenna with EBG Structure}

Once the antenna and the EBG structures have been designed, the next step is the integration of both resonant structures together in the same layer forming a uniplanar design [22]. As it has been already mentioned in the introduction, the resonance frequency of both structures is mutually influenced, and so depending on the frequency difference between them, and how the unit-cell arrangement around the patch antenna is, the resulting resonance frequency will change. The design of the patch antenna surrounded by 


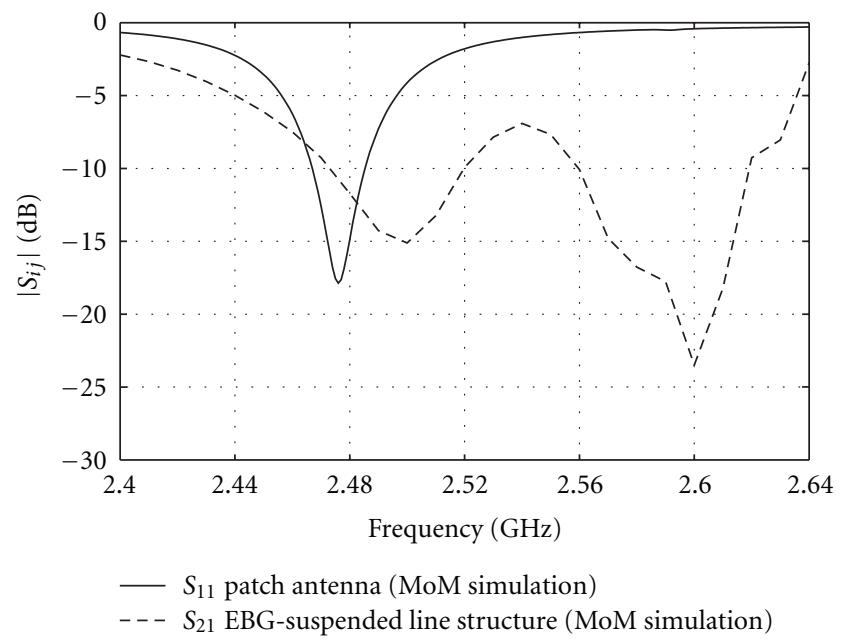

FIGURE 3: Resonances to be coupled in order to achieve bandwidth enhancement.

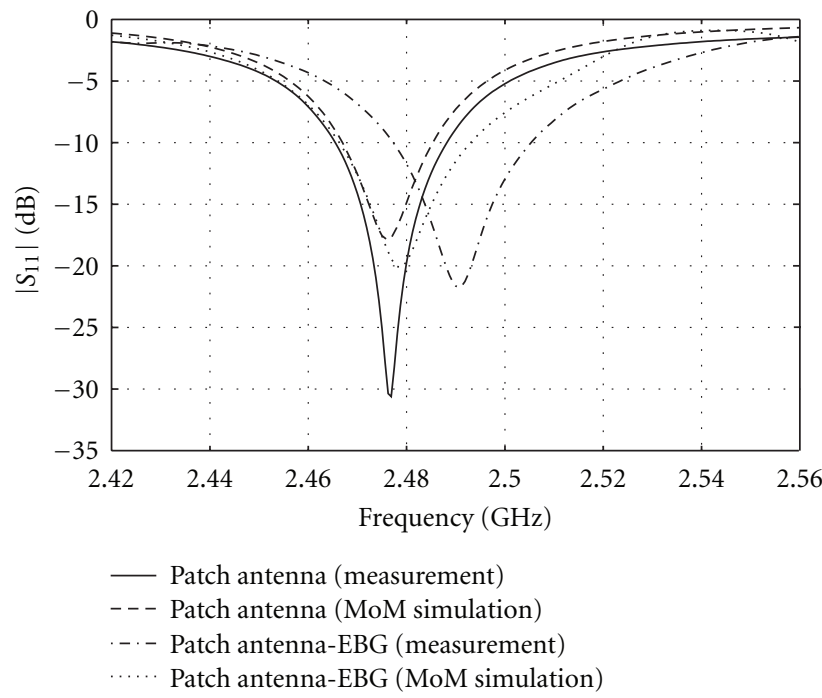

FIGURE 4: Simulation and measurement comparison between the patch antenna and the patch antenna-EBG prototypes.

one row EBG lattice has been carried out (Figure 1(b)). In FEM, due to the air-box size when small frequencies are involved (as in this case), the electric size of the problem to be solved is rather big. A proper mesh should take at least 10 (generally 20) tetrahedra per wavelength. Depending on the prototype's physical size, this could make the matrix of the linear equation system to become dense, which is not desirable in FEM and generally leads to longer computational times and increased memory requirements. However the matrix in MoM is dense, so this is not a problem, and thus this could be a better choice in general. The disadvantage of MoM is related to dielectric managements as they are considered infinite sized. Using MoM the mesh has been defined so that 20 cells per wavelength at $3 \mathrm{GHz}$ are taken which leads to 2460 rectangular cells and 6246 triangular cells with a matrix size of 12365 . After applying mesh reduction a matrix size of 5832 results. The simulation time for 81 frequency steps is 63 minutes in a 2-core Intel Xeon X5560 48 GB RAM (equivalent to 16 threads execution) server.

A prototype of the Patch antenna-EBG has been manufactured using laser micromachining. The return losses of the manufactured prototype have been measured and compared to those of the microstrip patch antenna (Figure 4). The principle of achieving bandwidth enhancement is based on coupling the resonances of the structures involved. As the patch antenna resonates at adjacent frequency band compared to the band-gap of the EBG lattice, a significant bandwidth enhancement of the prototype combining the two structures (Patch antenna-EBG) is obtained. As shown in Figure 4 the resulting bandwidth $(34 \mathrm{MHz})$ of the Patch antenna-EBG is $50 \%$ wider than the microstrip patch antenna's bandwidth $(23 \mathrm{MHz})$. Radiation pattern measurements of the prototypes have been carried out in anechoic chamber to complete their characterization. 


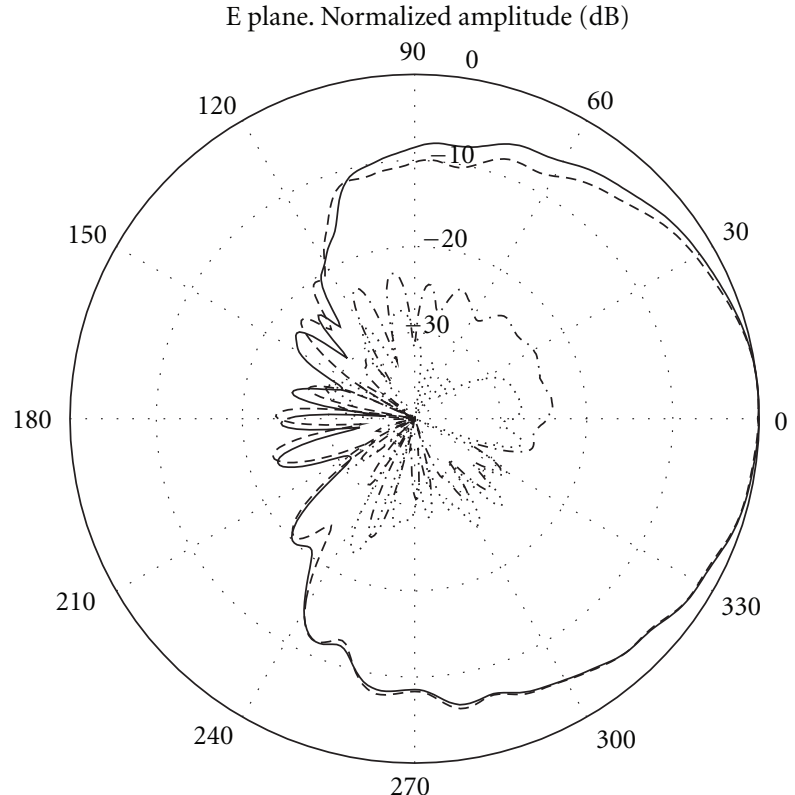

- Patch antenna, CP -. - Patch antenna, XP
- - Patch antenna-EBG, CP

(a)

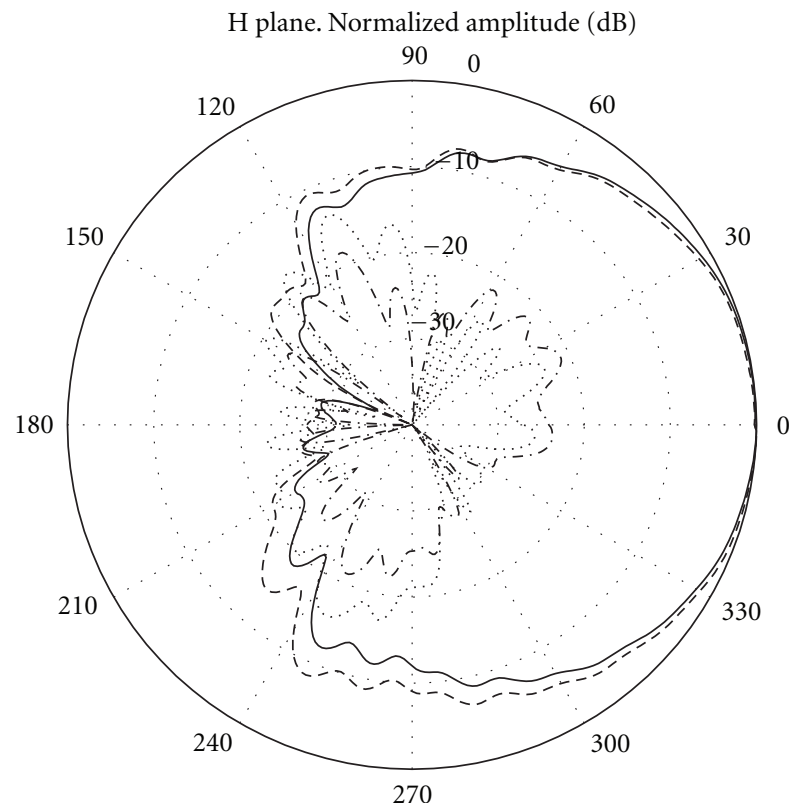

- Patch antenna, CP

- - - Patch antenna-EBG, CP

..... Patch antenna-EBG, XP

(b)

FIGURE 5: Patch antenna and patch antenna-EBG-measured radiation patterns.

TABLE 1: Comparison between the two prototypes.

\begin{tabular}{lccc}
\hline Prototype & Bandwidth $(\mathrm{MHz})$ & Directivity $(\mathrm{dB})$ & Gain $(\mathrm{dB})$ \\
\hline Patch antenna & 23 & 7.33 & 4.59 \\
Patch antenna-EBG & 34 & 7.50 & 4.61 \\
\hline
\end{tabular}

Radiation pattern cuts in the $\mathrm{E}$ and $\mathrm{H}$ planes of each manufactured prototype are plotted in Figure 5. Using an EBG structure to surround the patch antenna, the directivity increases due to the surface wave suppression (Table 1). In the case of placing the patch antenna in a frequency range outside the band-gap of the EBG structure, MoM simulations show that the unit cells have no influence in the radiation properties or in the bandwidth.

\section{Conclusions}

Bandwidth enhancement of microstrip patch antenna by means of EBG structure for RFID SHF $2.48 \mathrm{GHz}$ band has been presented. Using High-Performance computing electromagnetic methods the electrical characteristics of the resonant unit cell and the patch antenna have been evaluated both separated as well as combined in the same layer. The simulated and measured results are in good agreement due to precision of the methods (MoM and FEM) used in simulations. Both frequency domain techniques, MoM and FEM, can be used once the AMC is designed using FEM with PBCs. This is just an example of MoM and FEM techniques application to the design of antennas with metamaterials, but there are also other possible approaches, time domain based such as (Finite-difference time-domain) FDTD which could also be used.

There was reported a 50\% increase of the initial bandwidth. The patch antenna-EBG prototype presented in this paper has several advantages such as planar feature, compact size, and low dielectric losses. Neither via holes nor multilayer substrates are required, simplifying practical implementation and reducing its cost.

\section{Acknowledgments}

This work has been supported by the Ministerio de Ciencia e Innovación of Spain/FEDER under projects TEC200801638/TEC (INVEMTA) and CONSOLIDER-INGENIO CSD2008-00068 (TERASENSE), by the Gobierno del Principado de Asturias (PCTI)/FEDER-FSE under projects EQUIP08-06, FC09-COF09-12, EQUIP10-31, and PC10-06 (FLEXANT), by grant BP10-039, and by Cátedra TelefónicaUniversidad de Oviedo.

\section{References}

[1] R. Garg, I. Bhartia, I. Bahl, and A. Ittipiboon, Microstrip Antenna Design Handbook, Artech House, Boston, Mass, USA, 2001.

[2] A. Pirhadi, F. Keshmiri, M. Hakkak, and M. Tayarani, "Analysis and design of dual band high directivity EBG resonator antenna using square loop FSS AS superstrate layer," Progress in Electromagnetics Research, vol. 70, pp. 1-20, 2007. 
[3] E. Rajo-Iglesias, L. Inclán-Sánchez, and O. Quevedo-Teruel, "Back radiation reduction in patch antennas using planar soft surfaces," Progress in Electromagnetics Research Letters, vol. 6, pp. 123-130, 2009.

[4] Z. Duan, S. Qu, and Y. Hou, "Electrically small antenna inspired by spired split ring resonator," Progress in Electromagnetics Research Letters, vol. 7, pp. 47-57, 2009.

[5] F. Yang and Y. Rahmat-Samii, Electromagnetic Band-Gap Structures in Antenna Engineering, The Cambridge RF and Microwave Engineering Series, Cambridge, University Press, 2008.

[6] M. E. de Cos, F. Las-Heras, and M. Franco, "Design of planar artificial magnetic conductor ground plane using frequencyselective surfaces for frequencies below $1 \mathrm{GHz}$," IEEE Antennas and Wireless Propagation Letters, vol. 8, pp. 951-954, 2009.

[7] O. Luukkonen, C. R. Simovski, and S. A. Tretyakov, "Grounded uniaxial material slabs as magnetic conductors," Progress in Electromagnetics Research B, no. 15, pp. 267-283, 2009.

[8] H. Shaban, H. Elmikaty, and A. Shaalan, "Study the effects of electromagnetic band-gap (EBG) substrate on two patch microstrip antenna," Progress in Electromagnetics Research B, vol. 10, pp. 55-74, 2008.

[9] A. P. Feresidis, G. Goussetis, S. Wang, and J. C. Vardaxoglou, "Artificial magnetic conductor surfaces and their application to low-profile high-gain planar antennas," IEEE Transactions on Antennas and Propagation, vol. 53, no. 1, pp. 209-215, 2005.

[10] H. Mosallaei and K. Sarabandi, "Antenna miniaturization and bandwidth enhancement using a reactive impedance substrate," IEEE Transactions on Antennas and Propagation, vol. 52, no. 9, pp. 2403-2414, 2004.

[11] L. Akhoondzadeh-Asl, D. J. Kern, P. S. Hall, and D. H. Werner, "Wideband dipoles on electromagnetic bandgap ground planes," IEEE Transactions on Antennas and Propagation, vol. 55, no. 9, pp. 2426-2434, 2007.

[12] J. Liang and H. Y. D. Yang, "Radiation characteristics of a microstrip patch over an electromagnetic bandgap surface," IEEE Transactions on Antennas and Propagation, vol. 55, no. 6, pp. 1691-1697, 2007.

[13] F. Yang and Y. Rahmat-Samii, "Reflection phase characterizations of the EBG ground plane for low profile wire antenna applications," IEEE Transactions on Antennas and Propagation, vol. 51, no. 10, pp. 2691-2703, 2003.

[14] J. R. Sohn, K. Y. Kim, H. S. Tae, and J. H. Lee, "Comparative study on various artificial magnetic conductors for low-profile antenna," Progress in Electromagnetics Research, vol. 61, pp. 2737, 2006.

[15] S. Chaimool, K. L. Chung, and P. Akkaraekthalin, "Bandwidth and gain enhancement of microstrip patch antennas using reflective metasurface," IEICE Transactions on Communications, vol. E93-B, no. 10, pp. 2496-2503, 2010.

[16] D. Nashaat, H. A. Elsadek, E. A. Abdallah, M. F. Iskander, and H. M. Elhenawy, "Ultrawide bandwidth $2 \times 2$ microstrip patch array antenna using electromagnetic band-gap structure (EBG)," IEEE Transactions on Antennas and Propagation, vol. 59, no. 5, pp. 1528-1534, 2011.

[17] "ADS Momentum EM simulator tool," http://www.agilent .com/find/eesof.

[18] D. Sievenpiper, L. Zhang, R. F. J. Broas, N. G. Alexöpolous, and E. Yablonovitch, "High-impedance electromagnetic surfaces with a forbidden frequency band," IEEE Transactions on Microwave Theory and Techniques, vol. 47, no. 11, pp. 20592074, 1999.
[19] M. E. de Cos, Y. Álvarez, R. C. Hadarig, and F. Las-Heras, "Novel SHF-band uniplanar artificial magnetic conductor," IEEE Antennas and Wireless Propagation Letters, vol. 9, pp. 4447, 2010.

[20] A. Aminian, F. Yang, and Y. Rahmat-Samii, "In-phase reflection and EM wave suppression characteristics of electromagnetic band gap ground planes," in IEEE International Antennas and Propagation Symposium, vol. 4, pp. 430-433, June 2003.

[21] L. Yang, M. Fan, F. Chen, J. She, and Z. Feng, "A novel compact electromagnetic-bandgap (EBG) structure and its applications for microwave circuits," IEEE Transactions on Microwave Theory and Techniques, vol. 53, no. 1, pp. 183-190, 2005.

[22] M. E. de Cos, Y. Álvarez, and F. Las-Heras, "Enhancing patch antenna bandwidth by means of uniplanar EBG-AMC," Microwave and Optical Technology Letters, vol. 53, no. 6, pp. 1372-1377, 2011. 

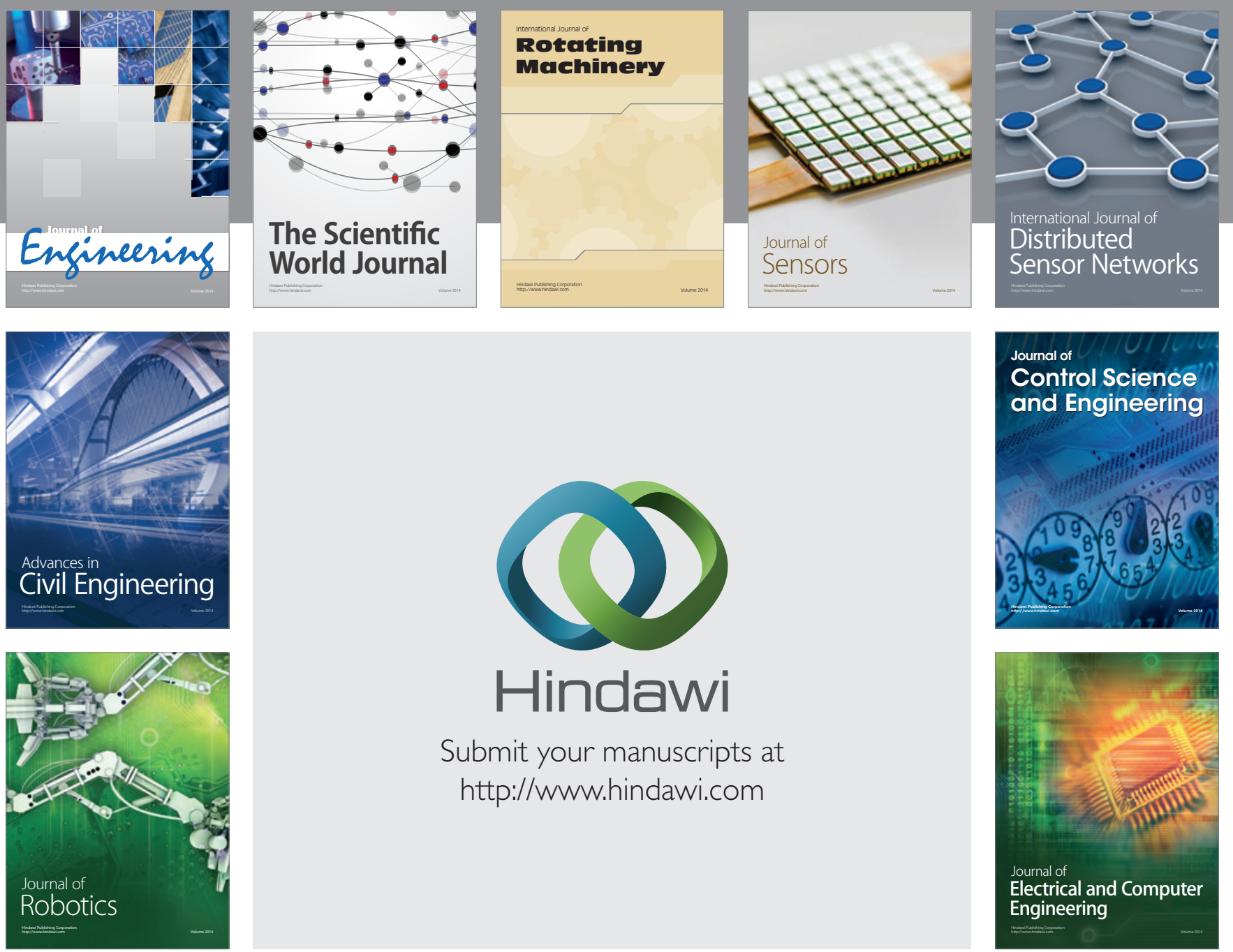

Submit your manuscripts at

http://www.hindawi.com
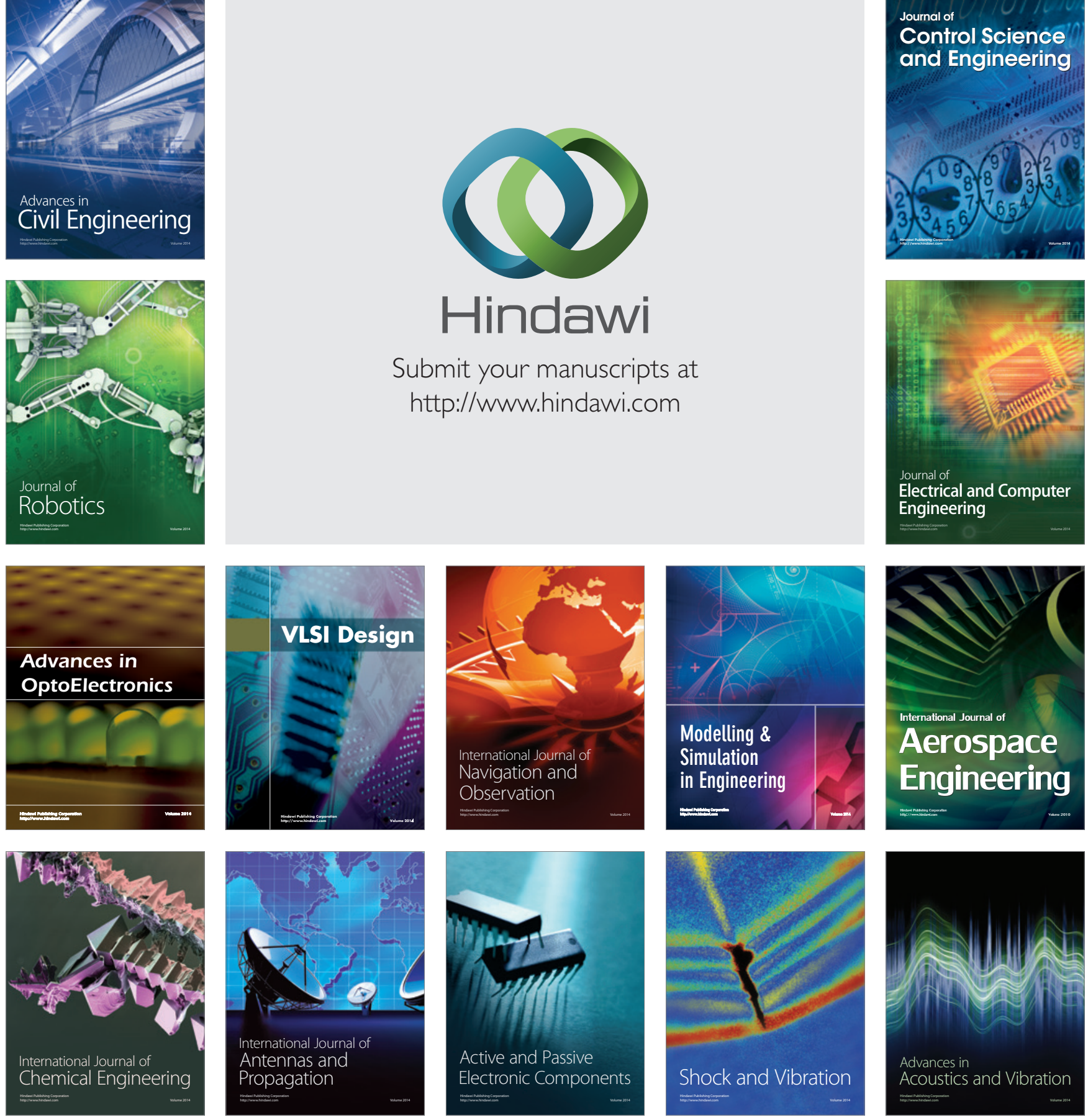